\title{
The Role of Genetic Mutation in Gene GBE1 In Induce Glycogen Storage Type IV Syndrome
}

\author{
Shahin Asadi* \\ Department of Medical Genetics, Iran \\ *Corresponding author: Shahin Asadi, Division of Medical Genetics and Molecular Pathology Research, Iran

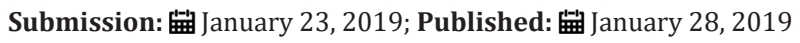

\begin{abstract}
Glycogen type IV storage syndrome is a hereditary genetic disorder caused by excessive accumulation of glycogen sugar complexes in the body's cells. The type of muscular nervous system is the most severe type of glycogen storage syndrome type IV, whose symptoms develop before birth and are usually fatal. Type IV glycogen storage syndrome is caused by gene mutations of GBE1, which is based on the short arm of chromosome number 3 as $3 \mathrm{p} 12.2$.
\end{abstract}

Keywords: Glycogen type IV storage syndrome; GBE1 gene; Metabolic disorder

\section{Case Report}

\section{Generalizations of type IV glycogen storage syndrome}

Glycogen type IV storage syndrome is a hereditary genetic disorder caused by excessive accumulation of glycogen sugar complexes in the body's cells. This accumulated glycogen is structurally abnormal and disrupts the function of some organs, tissues, especially the liver and muscles. There are five types of type IV glycogen storage syndrome that have different signs and symptoms [1].

\section{Symptoms of type IV glycogen storage syndrome}

The type of muscular nervous system is the most severe type of glycogen storage syndrome type IV, whose symptoms develop before birth and are usually fatal. In the muscular nervous system of the syndrome, excessive fluid can be created around the fetus

(polyhydramnios). Embryos affected by this type of type IV glycogen storage syndrome have stiffness after the birth, which can reduce their mobility. Neonates with type IV glycogen storage syndrome have a very weak muscle tone (muscle weakness) or severe muscular atrophy. These infants die at the same time of infancy due to weakness in the heart and respiratory muscles [2].

Congenital muscle type IV glycogen storage syndrome is not usually known prior to birth, but its symptoms develop at the beginning of the birth. Damaged babies have severe muscular hypotension that affects the muscles needed for respiration. These infants often experience obstructive cardiomyopathy, in which the heart is unable to pump blood properly. It is worth noting that infants with congenital type IV glycogen storage syndrome typically live only a few months [3].
Progressive liver failure is the most common form of glycogen storage type IV syndrome. In the first months of life, damaged babies experience weight gain, growth failure, and large liver (hepatomegaly). Children with this type IV glycogen storage syndrome develop liver disease called cirrhosis of the liver, which is often irreversible Figure 1. In addition, high blood pressure in the vein that transports the blood to the liver (hypertensive portal portal) and abnormal fluid production in the abdominal cavity (ascites) can also occur in these children. Children 1 to 2 years of age with this syndrome have poor muscle tone (hypotonia) and often die in childhood due to liver failure [4].

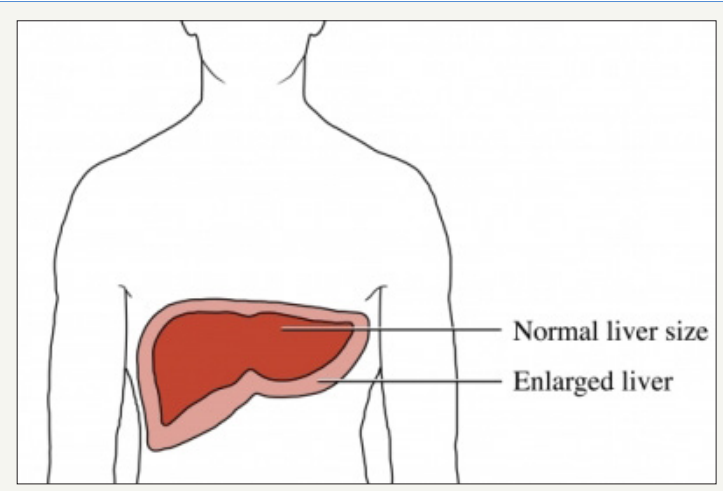

Figure 1: Schematic of the normal liver in versus of the large liver (hepatomegaly).

Non-progressive liver disorder in type IV glycogen storage syndrome has many similar characteristics to the progressive liver, but liver disease in this disorder is not severe. In the nonprogressive type of liver, hepatomegaly and hepatic dysfunction usually appear in childhood, but these children typically do not 
cause liver cirrhosis. Patients with this type 4 glycogen storage syndrome also have muscle weakness (hypothyroidism) and muscle weakness (myopathy). It should be noted that most children with non-progressive liver glycogen storage syndrome reach puberty, although life expectancy in these individuals varies depending on the severity of the symptoms and symptoms [4].
Childhood Neonatal muscle skeletal type IV glycogen storage syndrome is revealed at the end of childhood and is characterized by myopathy and obstructive cardiomyopathy. The severity of this type of glycogen storage syndrome is very different Figure 2. Some people with mild impairment (mild), while others with severe cardiomyopathy die in early adulthood [4].

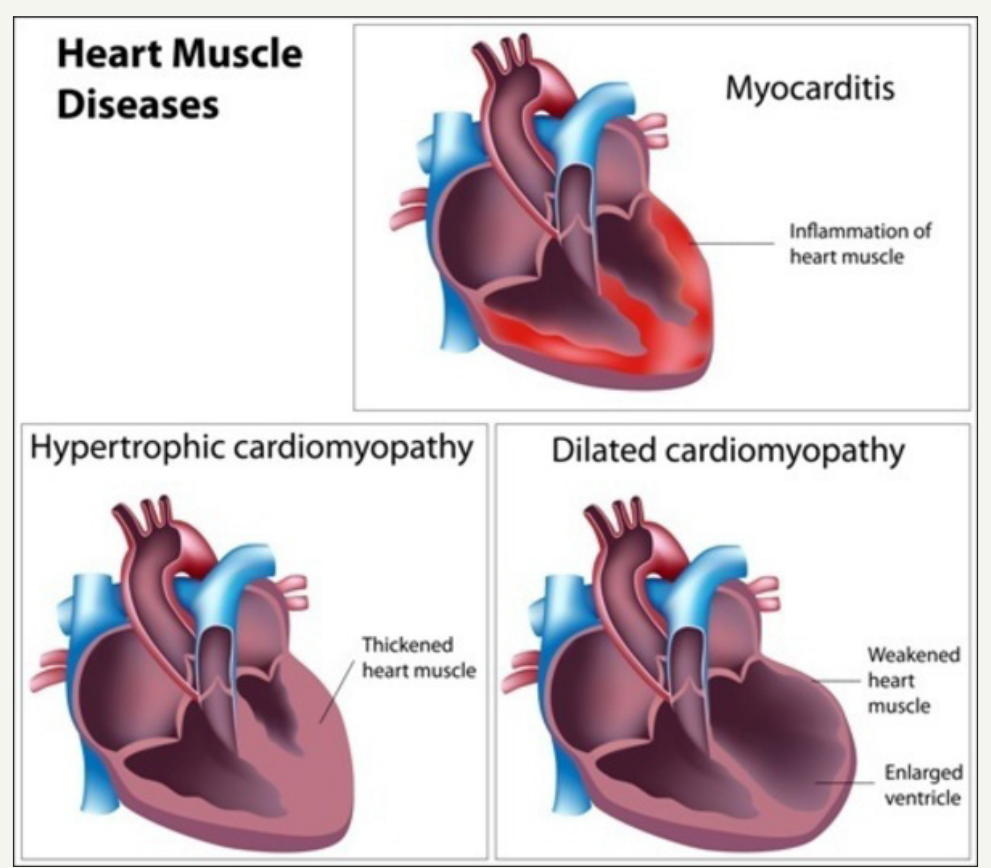

Figure 2: Schematic of heart disorders in type IV glycogen storage syndrome.

\section{Etiology of type IV glycogen storage syndrome}

Type IV glycogen storage syndrome is caused by gene mutations of GBE1, which is based on the short arm of chromosome number 3 as $3 p 12.2$. This gene provides instructions for the synthesis of the branched-chain enzyme glycogen. This enzyme is involved in glycogen production, which is the main source of stored energy in the body. The GBE1 gene mutations that cause type IV glycogen storage syndrome result in deficiency of the glycogen branching enzyme, and thus the glycogen does not form properly. Abnormal glycogen molecules accumulate in the cells of the body called polyglucosan, resulting in cell damage and death. Polyglucosan substances are accumulated throughout the cells of the body, but liver cells and muscle cells in the type IV glycogen storage syndrome are heavily influenced by these objects. Glycogen accumulation in the liver leads to hepatomegaly and causes liver function impairment Figure 3. Therefore, the inability of the muscle cells to degrade glycogen for energy leads to muscle weakness [5].

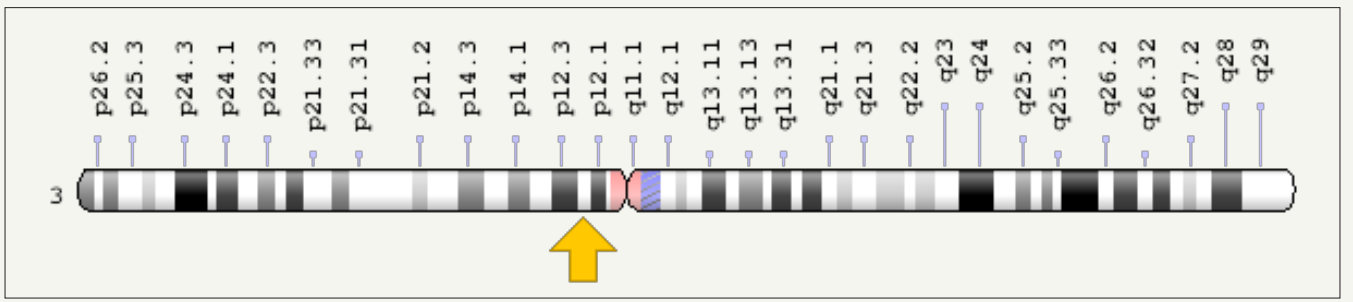

Figure 3: Schematic view of chromosome number 3, in which the GBE1 gene is based on the short arm of this chromosome as 3p12.2.

In general, the severity of glycogen type IV storage syndrome is related to the amount of glycogen functional branch enzymes. People with type IV muscular glycogen storage syndrome produce less than $5 \%$ of the branched-chain enzyme glycogen in their cells, while those with type IV glycogen storage syndrome have a childhood of about $20 \%$ The enzyme produces glycogen in their cells, and other types of the syndrome usually produce 5 to 20 percent of the branched-chain glycogen enzymes [5]. Type IV glycogen storage syndrome follows an autosomal recessive hereditary pattern. Therefore, in order to create this syndrome, two versions of the genetically engineered GBE1 gene (one parent and one mother) are needed and the chance of having a child with type 
IV glycogen storage syndrome in an autosomal recessive state, for each probable pregnancy, is $25 \%$ is [6].

\section{Frequency of type IV glycogen storage syndrome}

Type IV glycogen storage syndrome is a very rare hereditary genetic disorder with its worldwide prevalence rate of about 1 in 600,000 to 1 in 800,000 live births Figure 4. Type IV glycogen storage syndrome accounts for about $3 \%$ of all cases of glycogen storage disease [6].

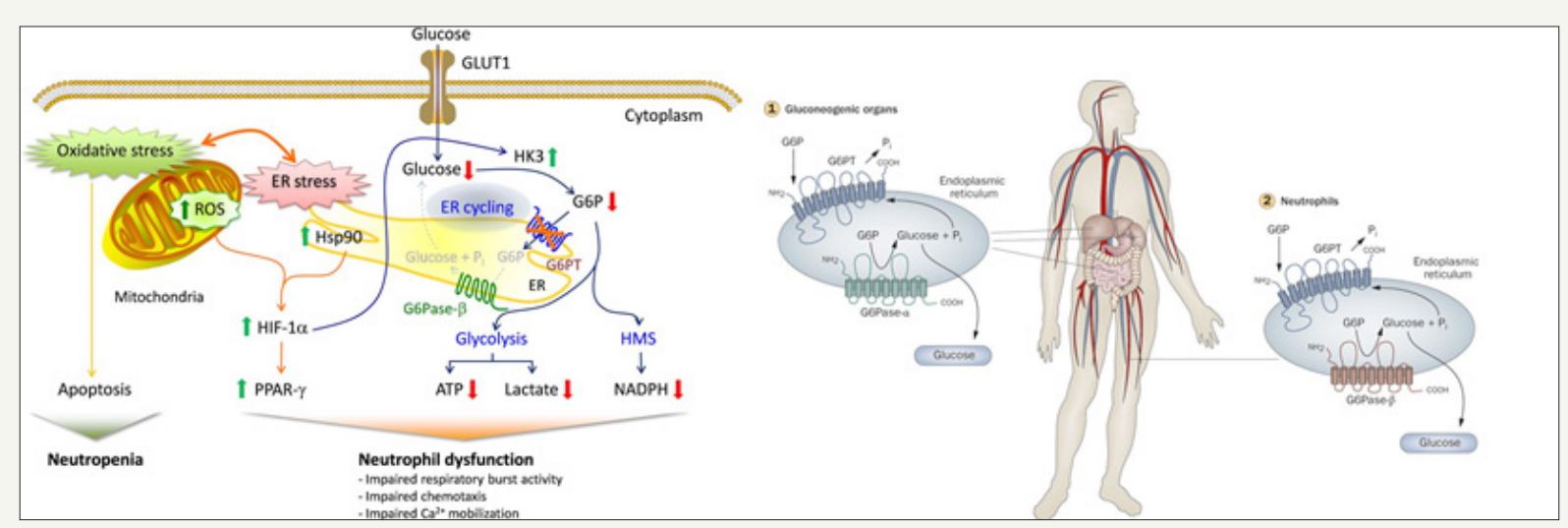

Figure 4: A schematic of the biochemical pathway of glucose in the cell.

\section{Diagnosis of type IV glycogen storage syndrome}

Type IV glycogen storage syndrome is diagnosed based on the clinical, clinical and physical findings of the patients and some pathological tests. The most accurate diagnostic method for this syndrome is the molecular genetic testing of the GBE1 gene to investigate the presence of possible mutations Figure 5. Prenatal diagnosis is also possible by using the PGD technique and amniocentesis fluid or by sampling the embryo's chorionic pelvis [7].

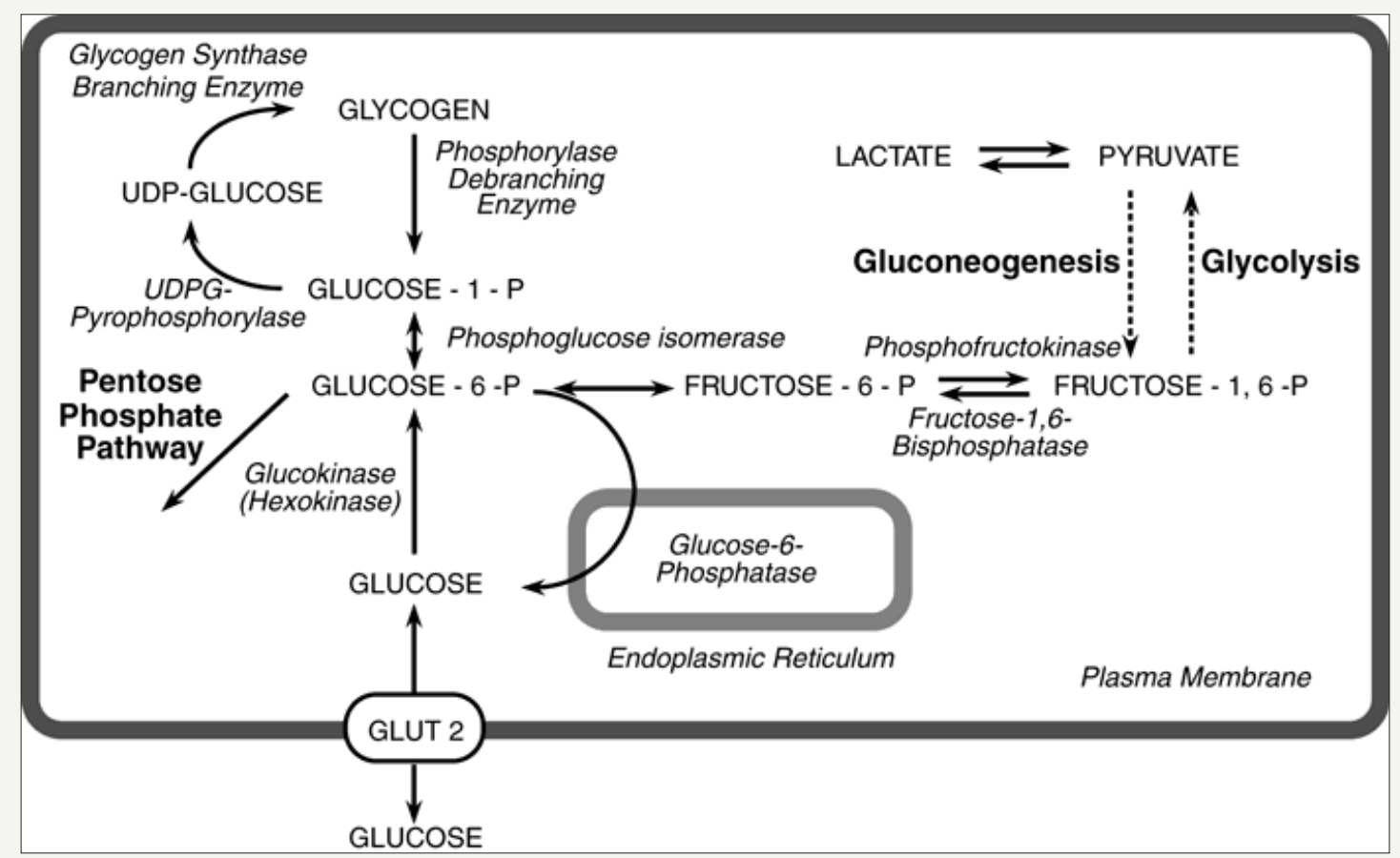

Figure 5: Schematic of the pathway for glucose to glycogen conversion in the cell membrane. Treatment routes for type IV glycogen storage syndrome

\section{Treatment routes for type IV glycogen storage syndrome}

The management and management of type IV glycogen storage syndrome is symptomatic and supportive. Treatment may be done by a team of experts including pediatrician, nephrologist, liver, gastroenterologist, cardiologist, clinical specialist, orthopedic specialist, dietitian, neurologist, pediatrician and other health care professionals. There is no definite treatment for this syndrome and all clinical measures are needed to reduce the suffering of the infected person Figure 6. Genetic counseling is also needed for all parents who want a healthy baby [7]. 


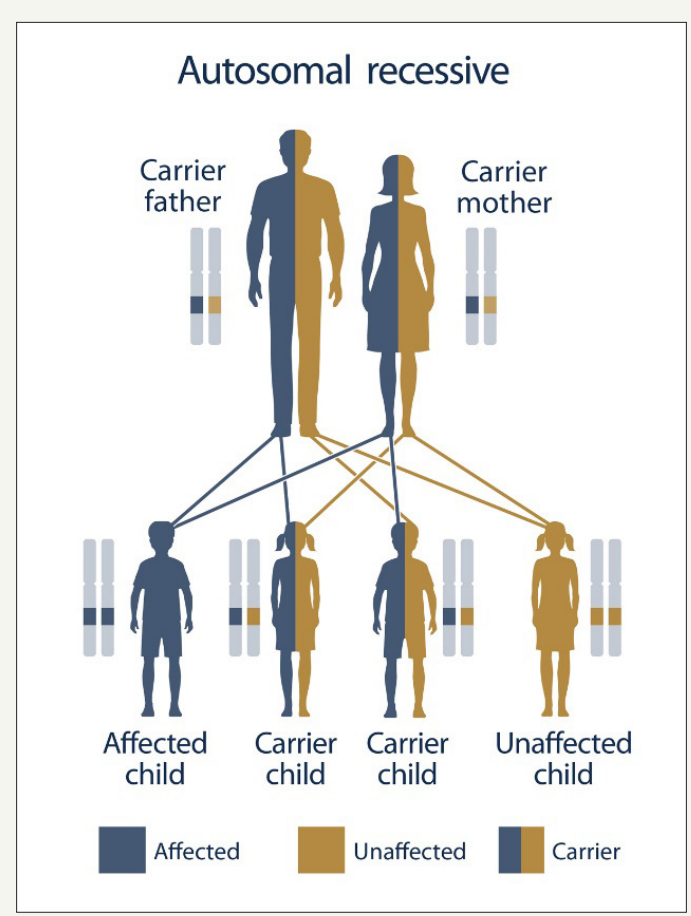

Figure 6: A schematic view of an autosomal recessive hereditary pattern that follows the type IV glycogen storage syndrome.

\section{Discussion and conclusion}

Glycogen type IV storage syndrome is a hereditary genetic disorder caused by excessive accumulation of glycogen sugar complexes in the body's cells. The type of muscular nervous system is the most severe type of glycogen storage syndrome type IV, whose symptoms develop before birth and are usually fatal. Type IV glycogen storage syndrome is caused by gene mutations of GBE1, which is based on the short arm of chromosome number 3 as $3 p 12.2$. There is no definite treatment for this syndrome and all clinical measures are needed to reduce the suffering of the infected person.

\section{References}

1. Assereto S, Diggelen OP, Diogo L, Morava E, Cassandrini D, et al. (2007) Null mutations and lethal congenital form of glycogen storage disease type IV. Biochem Biophys Res Commun 361(2): 445-450.
2. Bruno C, Cassandrini D, Assereto S, Akman HO, Minetti C, et al. (2007) Neuromuscular forms of glycogen branching enzyme deficiency. Acta Myol 26(1): 75-78.

3. Bruno C, Diggelen OP, Cassandrini D, Gimpelev M, Giuffrè B, et al. (2004) Clinical and genetic heterogeneity of branching enzyme deficiency (glycogenosis type IV). Neurology 63(6): 1053-1058.

4. Burrow TA, Hopkin RJ, Bove KE, Miles L, Wong BL, et al. (2006) Nonlethal congenital hypotonia due to glycogen storage disease type IV. Am J Med Genet A 140(8): 878-882.

5. Fernandez C, Halbert C, Paula AM, Lacroze V, Froissart R, et al. (2010) Non-lethal neonatal neuromuscular variant of glycogenosis type IV with novel GBE1 mutations. Muscle Nerve 41(2):269-271.

6. Magoulas PL, Hattab AW, Roy A, Bali DS, Finegold MJ, et al. (2012) Diffuse reticuloendothelial system involvement in type IV glycogen storage disease with a novel GBE1 mutation: a case report and review. Hum Pathol 43(6): 943-951.

7. Tay SK, Akman HO, Chung WK, Pike MG, Muntoni F, et al. (2004) Fatal infantile neuromuscular presentation of glycogen storage disease type IV. Neuromuscul Disord 14(4): 253-260.

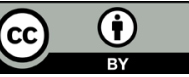

Creative Commons Attribution 4.0 International License

For possible submissions Click Here

\section{Submit Article}

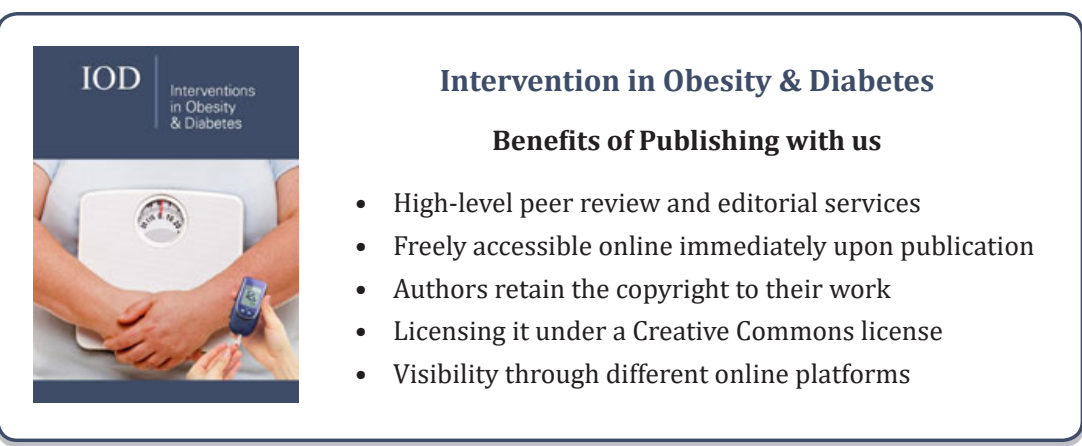

